\title{
Development and validation of humming process to increase heart rate variability $\&$ attention for college students
}

\author{
Irani Fariburz $Z^{1 *}$, Trivedi Gunjan $\mathrm{Y}^{2}$, Sinha Neeta ${ }^{3}$
}

\section{ABSTRACT}

Aim: The objective of the study was to compare the impact of daily $10 \mathrm{~min}$ humming practice for one week on heart-brain coherence and attention on college students. Methodology: The evidence indicates that increased heart-brain coherence facilitates better emotional regulation and thereby increases attention. Simple humming practice was identified as a method to increase the heart-brain coherence. Each student was assigned randomly to an experimental group or control group. The experimental group $(n=15)$ performed 10 -minutes humming daily for 7 days and the control group $(n=15)$ was not asked to do anything. 10-minute humming was simplified Bhramari practice with total breath duration of 13 seconds based on short training. SDMT (Symbol Digit Modalities Test) and Heart Brain Coherence (using Emwave Pro Device by HeartMath Inc.) was conducted for both the groups on Day 1 and Day 7. Statistical analysis was conducted using student-t test to understand the results. Results: For the experimental group, there was statistically significant improvement in both "Coherence" and "SDMT" score on Day 7 (compared to Day 1, $\mathrm{p}<.05$ ). For control group, the change in both parameters was not statistically significant. Conclusions: The results confirm that a short duration humming practice just for one week can increase the heart-brain coherence and Heart Rate Variability and has a significant impact on increasing attention amongst the college students. These findings can be leveraged to facilitate better concentration and potentially increased performance for college students.

Keywords: Heart Rate Variability, HRV, Coherence, Humming, Attention, College Students

Key Messages: Simple humming increases Heart Rate Variability (HRV). Increased HRV influences emotional regulation, reduces stress and increases attention and focus. This study demonstrated that compared to the control group (which did not do any humming), the experimental group (practiced 10 mins humming daily for 7 days) demonstrated increased HRV and a statistical increase in SDMT scores.

\footnotetext{
${ }^{1}$ B.A. (Hons) Psychology, School of Liberal Studies, PDPU, Gujarat, India

${ }^{2}$ Cofounder, Society for Energy \& Emotions, Wellness Space, Ahmedabad, India

${ }^{3}$ Assistant Professor, School of Liberal Studies, PDPU, Gujarat, India

*Responding Author
}

Received: October 12, 2020; Revision Received: February 14, 2021; Accepted: March 03, 2021

(C) 2021, Irani F. Z., Trivedi G. Y. \& Sinha N.; licensee IJIP. This is an Open Access Research distributed under the terms of the Creative Commons Attribution License (www.creativecommons.org/licenses/by/2.0), which permits unrestricted use, distribution, and reproduction in any Medium, provided the original work is properly cited. 
$\mathrm{D}$ ue to the increasing prevalence of cell phone usage and a general increase in screen time, there is a marked decrease in the attention span, especially in young adults and students $^{\mathrm{i}}{ }^{\text {ii }}$. It gets challenging for students to concentrate for longer durations of time as there are consecutive lectures held for around an hour each and this leads to mental exhaustion. Studies have shown that guided breathing, exercising help in increasing focus and cognition through changes in beat-to-beat variations in the heart's signals (Heart Rate Variability-HRV) $)^{\mathrm{iii}}$. The increase in HRV, influenced by coherence between heart and the brain signals, balances the nervous system and increases emotional regulation ${ }^{\mathrm{iv}}$.

Studies have shown that increased HRV (using techniques such as slow breathing or HRV biofeedback, chanting or meditation) is linked to better cognitive function (attention \& focus) $)^{\mathrm{v}}$ vi vii. This study explored if regular practice of humming (slow breathing, i.e. slow inhalation followed by longer exhalation while making a humming sound during exhalation) could increase HRV and thereby increase the focus and attention. This was based on a small experiment when we found that humming generates significant increase in heart's oscillations (HRV) and hence it was used as a technique to increase the HRV.

\section{Heart Rate Variability}

Heart Rate Variability (HRV) refers to the successive interval between two heart beats (also known as NN or RR intervals). It is indicative of the sympathetic and parasympathetic activity of the Autonomic Nervous System (ANS). High HRV is seen as a sign of lower anxiety levels and better emotional regulation. ${ }^{\text {viii }}$ Low HRV represents prevalence of higher sympathetic ANS activity and high HRV indicates higher parasympathetic activity ${ }^{\text {ix }} \mathrm{x} \times \mathrm{xi}$. Decreased HRV indicates an imbalance in the ANS which helps serve as a non-invasive indicator of Cardio Metabolic Diseases (CMDs) xii xiii. Research shows that HRV is significantly associated with the blood flow in the ventromedial prefrontal region of the brain and the Amygdala. ${ }^{\text {xiv }}$ In both young and old adults higher HRV was associated with medial prefrontal cortex and amygdala connectivity ${ }^{\mathrm{xv}} \mathrm{xvi}$. The Prefrontal cortex is said to be related to higher attention and emotion regulation ${ }^{\text {xvii xviii xix } x x}$.

As discussed earlier, previous studies have demonstrated that chanting and HRV biofeedback increase attention ${ }^{x x i}$ xxii. However, the research in the area explored longer duration sessions (beyond 15 minutes) and over several weeks. This study explored if a short daily practice (10 minutes) for just one week can influence HRV and increase attention and focus. Moreover, the process of making humming sound has broader implications and does not involve any religious mantra and could have a much broader acceptance, especially amongst the students.

\section{METHODOLOGY}

\section{Participants and Design}

The study was conducted at an educational institute and the undergraduate students were recruited to participate in a week-long study. The participants were randomly divided into two groups (experimental \& control) without informing them about the other group. The written consent was taken from them to be a part of this study. The students on any regular medication or with chronic disease or recent surgery were excluded from the study. The average age of the subjects in the experimental group was 20.33 and the standard deviation was \pm 1.11 , for the control group the average age was 20.47 and the standard deviation was \pm 0.99 . There were 6 males and 9 females in the control group and 5 males and 10 females in the experimental group. 


\section{Protocol}

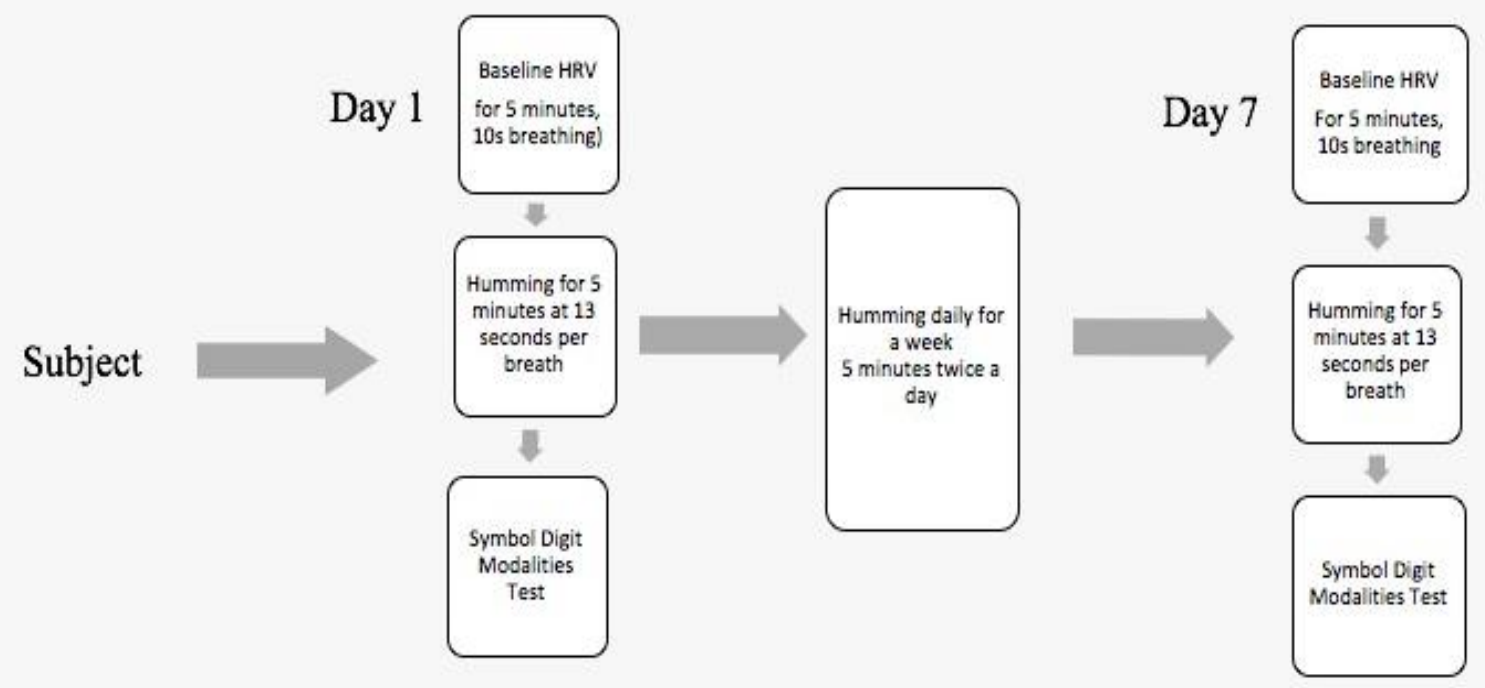

Figure 1.1: The study design of the experimental group

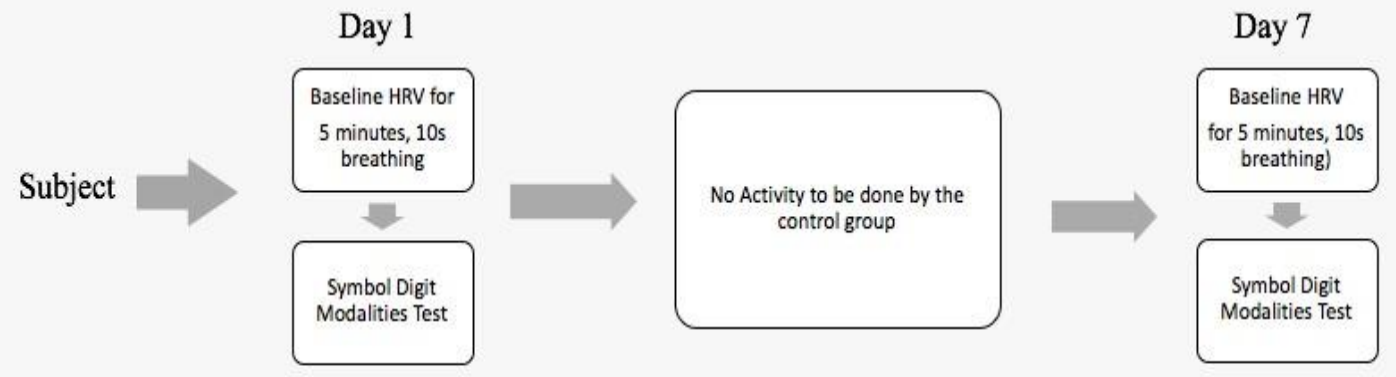

\section{Figure 1.2 The study design of the control group}

The protocol is shown in Figure 1.1 and 1.2. Each student was randomly assigned to either the experimental group or the control group. For both the groups the experiment was done only with one student at a time. The experiment was conducted in an air-conditioned room where the temperature was maintained at 25 degree centigrade. The measurements were conducted between 10:00 AM to 1:00 PM and the participant did not consume any tea or coffee two hours before the measurements.

\section{Measurement tools}

The symbol-digit modalities test (SDMT) is a symbol substitution neuropsychological test that examines a person's attention, concentration and speed of processing. ${ }^{\text {xxii }}{ }^{x x i v}$ SDMT test 
scores do not vary much by age, education, gender and income groupings in healthy individuals $^{\mathrm{xxv}}$. Hence, for this study, the written version of the SDMT test was used to measure attention and focus (concentration, speed of processing) for college students. The intermediate measure, HRV, was used based on the data provided by Emwave Pro (Achievement and Coherence parameters). These two parameters based on the output of the Emwave Pro and have been used in several studies as measures of HRV ${ }^{\text {xxvi xxvii xxviii. }}$

\section{The experimental group (Humming)}

This group practiced 10 mins humming daily for one week $(n=15)$. As shown in figure 1.1 after measuring the subject's attention, focus and HRV on Day 1, they were requested to practice simple humming for 10 mins daily for one week. Every day they would practise the humming for 5 minutes in the morning and 5 minutes in the night at the same time every day. After one week, the attention, focus and HRV measurements were repeated. Attention and focus was measured using Symbol Digit Modalities Test (SDMT) and HRV was measured using two parameters from Emwave Pro device ${ }^{\mathrm{xxix}}$ (Achievement and Coherence). Breathe android application ${ }^{\mathrm{xxx}}$ (made by Jatra, Inc) was used to ensure consistency for each participant. Day 1 and Day 7 measurements for HRV were conducted while the students perform a slow guided breathing exercise of 10 seconds long breath for 5 minutes followed by 13 seconds long simple humming for 5 minutes with the help of the "Breathe" mobile application. HRV was measured using Emwave Pro device (Heartmath, Inc) and Coherence and Achievement parameters from the software were noted into an Excel document $^{\mathrm{xxxi}}$.Students were asked to practise this simple humming for 5 minutes each in the morning and evening for 7 days. On the last day they were again tested for attention and focus using the SDMT and then they were again asked to perform the breathing exercise and simple humming while their Heart Brain coherence was measured.

\section{The control group}

As shown in figure 1.2 the control group was not introduced to any humming practice and were not aware of the activities performed by the experimental group. This group was also tested for attention and focus using the SDMT and their Heart Brain Coherence and Achievement was measured for 5-minute paced breathing of 10 seconds on the first and seventh day. They were not asked to do any specific activity for those 7 days.

\section{Data Analysis}

Each group's data was tabulated in an Excel document with scores of SDMT and Coherence and Achievement numbers from Emwave Pro device. Summary of the data showing mean and standard deviation for each group is shown in Table 1. Statistical analysis for Student's T-Test was conducted using Excel feature and p-value is also highlighted in the table.

The SDMT is a test in which the subject substitutes the digit corresponding to a symbol and they are scored on the number of correct substitutions done in a minute. It measures

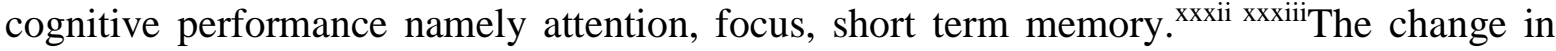
the SDMT scores was analysed and Student's T-Test was used to check for validity of the data.

\section{RESULTS}

The results confirmed a statistically significant improvement in SDMT Score in the Experiment group $(n=15)$ after daily humming practice of 10 minutes duration for one week based on Student's T-Test with a p-value of $0.00(<0.05)$. However, for control group 
$(n=15)$ there no significant change in the SDMT score ( $\mathrm{p}$-value $=0.4,>0.05)$. Table 1.1 captures the SDMT scores of both groups before and after the intervention.

Table 1.1 Change in SDMT scores for both groups and their standard deviation

\begin{tabular}{|l|l|l|l|l|l|}
\hline \multicolumn{1}{|c|}{ SDMT Score } & \multicolumn{1}{|c|}{ Before } & \multicolumn{1}{c|}{ After } & \multicolumn{1}{c|}{ Before } & \multicolumn{1}{c|}{ After } & p-Value \\
\hline $\begin{array}{l}\text { Experiment } \\
\text { Group* }\end{array}$ & 52.00 & 58.80 & 10.04 & 11.50 & 0.00 \\
\hline Control Group & 57.73 & 57.40 & 8.76 & 8.25 & 0.40 \\
\hline
\end{tabular}

For control group, the paced breathing achievement and coherence scores did not have any statistically significant changes ( $\mathrm{p}$-value $=0.33$ and 0.24 respective). Therefore, it can be concluded that the HRV for this group did not change significantly during the week and this could have resulted in no change in their SDMT scores (Table 1.2). It is important to add here that the experimental design ensured the control group did not do any humming or any other paced breathing activity during the week since that could have increased their HRV and influenced the SDMT score.

Table 1.2 Change in HRV parameters for the control group

\begin{tabular}{|l|l|l|l|l|l|l|}
\hline \multicolumn{1}{|c|}{ Measure } & \multicolumn{1}{c|}{ Mean } & \multicolumn{3}{c|}{ SD } & \\
\hline $\begin{array}{l}\text { Control Group } \\
\text { Achievement }\end{array}$ & Humming & Before & After & Before & After & p-Value \\
\hline $\begin{array}{l}\text { Control Group } \\
\text { Achievement }\end{array}$ & NA & NA & NA & NA \\
\hline $\begin{array}{l}\text { Control Group } \\
\text { Coherence } \\
\text { Breathing } \\
\text { Control Group - } \\
\text { Coherence }\end{array}$ & Humming & NA & NA & NA & NA & NA \\
\hline
\end{tabular}

For experiment group, the achievement score increased for both the humming and paced breathing activities in a statistically significant manner $(\mathrm{p}=0.04$ and 0.05 respectively, Table 1.3). Coherence for humming (the activity they performed for one week) increased in a statistically significant manner $(\mathrm{p}=0.04)$ for the experiment group, however the breathing coherence score showed an increase but it was not statistically significant $(\mathrm{p}=0.11)$

Table 1.3 Change in HRV parameters for the experimental group

\begin{tabular}{|l|l|l|l|r|r|r|}
\hline & \multicolumn{3}{|l|}{ Mean } & \multicolumn{1}{l|}{ SD } & p-Value \\
\hline Measure & Activity & Before & After & Before & After & p-V \\
\hline $\begin{array}{l}\text { Experimental Group - } \\
\text { Achievement* }\end{array}$ & Humming & 199.47 & 234.87 & 96.56 & 70.16 & 0.04 \\
\hline $\begin{array}{l}\text { Experimental Group - } \\
\text { Achievement* }\end{array}$ & $\begin{array}{l}\text { Paced } \\
\text { Breathing }\end{array}$ & 145.71 & 178.50 & 94.10 & 107.89 & 0.05 \\
\hline $\begin{array}{l}\text { Experimental Group - } \\
\text { Coherence }\end{array}$ & $\begin{array}{l}\text { Paced } \\
\text { Breathing }\end{array}$ & 2.72 & 3.18 & 1.53 & 1.83 & 0.11 \\
\hline $\begin{array}{l}\text { Experimental Group - } \\
\text { Coherence* }\end{array}$ & Humming & 3.47 & 4.09 & 1.67 & 1.25 & 0.04 \\
\hline
\end{tabular}

(c) The International Journal of Indian Psychology, ISSN 2348-5396 (e)| ISSN: 2349-3429 (p) | 247 


\section{Development and validation of humming process to increase heart rate variability \& attention for college students}

The results indicate that the prescribed daily humming may have contributed to the increase of humming scores for "Coherence" and "Achievement" for the experiment group. The group also increased the "Achievement" score for breathing while the "Coherence" for breathing did not increase in a statistically significant manner. This increase in coherence may have contributed to increased cognition (attention) as demonstrated by the increase in the SDMT scores.

\section{DISCUSSION}

The study has demonstrated that a short practice of 10 minutes daily for just one week could increase the attention and focus in the experiment group as demonstrated by increase in the SDMT score. This increase was clearly not observed in the control group. The increase in attention and focus is consistent with earlier findings however, the unique insight from this study is that a short duration practice for a week also has a positive impact on cognition. A previous study ${ }^{\text {xxiv }}$ in this area used Gayatri Mantra Chanting (daily 10 minutes) for 5 days. Another study ${ }^{\mathrm{xxx}}$ used biofeedback training for basketball players which had them breathe at their resonant frequency and showed improvement in HRV, response time, concentration etc. They were asked to practise for 20 minutes daily for 10 days. Compared to that, this study leveraged a simpler and more neutral method to increase HRV in a short time by introducing the participants to humming.

The findings can be incorporated into a routine where students can pursue this technique daily during college days to enhance their focus and attention. It does not require any external help so there is no dependence on any external device or guidance after a simple training is completed. The limitation is that this work requires self-discipline which may prevent a broad-based usage unless the individual chooses to prioritize this over other work. Future work in this could explore a larger sample size with different demographics (e.g. senior citizens, corporate employees). Future work can involve studies that focus on comparison between humming and breathing.

\section{CONCLUSION}

The results confirm that a short duration humming practice just for one week to increase the heart-brain coherence has a significant impact on increasing the attention amongst the college students. The increase in coherence and cognition did not happen in the control group that did not pursue the humming practice. These findings can be leveraged to increase concentration and potentially increased performance for college students. The future work in this area should explore different demographics with a larger sample size and could also compare humming and breathing as an intervention.

\section{REFERENCES}

\footnotetext{
${ }^{\mathrm{i}}$ Swing, E. L., Gentile, D. A., Anderson, C. A., \& Walsh, D. A. (2010). Television and video game exposure and the development of attention problems. Pediatrics, 126(2), 214221.

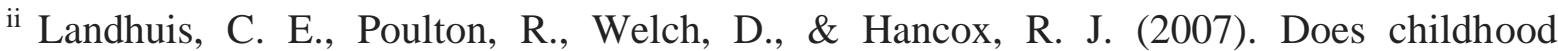
television viewing lead to attention problems in adolescence? Results from a prospective longitudinal study. Pediatrics, 120(3), 532-537.
} 
iii Luque-Casado, A., Zabala, M., Morales, E., Mateo-March, M., \& Sanabria, D. (2013). Cognitive performance and heart rate variability: the influence of fitness level. PloS one, 8(2), e56935

iv Mather, M., \& Thayer, J. F. (2018). How heart rate variability affects emotion regulation brain networks. Current opinion in behavioral sciences, 19, 98-104.

${ }^{v}$ Forte, G., \& Casagrande, M. (2019). Heart rate variability and cognitive function: a systematic review. Frontiers in neuroscience, 13, 710.

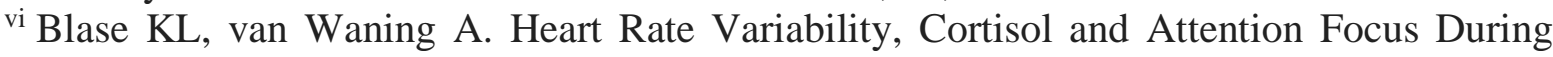
Shamatha Quiescence Meditation. Appl Psychophysiol Biofeedback. 2019 Dec;44(4):331-342. doi: 10.1007/s10484-019-09448-w. PMID: 31485894.

vii Mather, M., \& Thayer, J. F. (2018). How heart rate variability affects emotion regulation brain networks. Current opinion in behavioral sciences, 19, 98-104.

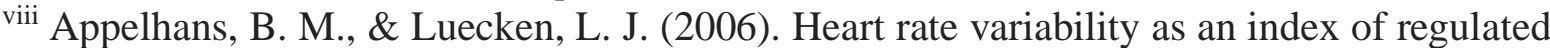
emotional responding. Review of general psychology, 10(3), 229-240.

${ }^{\text {ix }}$ Chalmers, J. A., Quintana, D. S., Abbott, M. J., \& Kemp, A. H. (2014). Anxiety disorders are associated with reduced heart rate variability: a meta-analysis. Frontiers in psychiatry, 5, 80 .

${ }^{x}$ Kemp, A. H., \& Quintana, D. S. (2013). The relationship between mental and physical health: insights from the study of heart rate variability. International Journal of Psychophysiology, 89(3), 288-296.

${ }^{x i}$ Shaffer, F., McCraty, R., \& Zerr, C. L. (2014). A healthy heart is not a metronome: an integrative review of the heart's anatomy and heart rate variability. Frontiers in psychology, 5, 1040.

xii Trivedi, G. Y., Saboo, B., Singh, R. B., Maheshwari, A., Sharma, K., \& Verma, N. (2019). Can decreased heart rate variability be a marker of autonomic dysfunction, metabolic syndrome and diabetes?. Journal of Diabetology, 10(2), 48.

xiii Brosschot, J. F., Van Dijk, E., \& Thayer, J. F. (2007). Daily worry is related to low heart rate variability during waking and the subsequent nocturnal sleep period. International journal of psychophysiology, 63(1), 39-47.

xiv Thayer, J. F., Åhs, F., Fredrikson, M., Sollers III, J. J., \& Wager, T. D. (2012). A metaanalysis of heart rate variability and neuroimaging studies: implications for heart rate variability as a marker of stress and health. Neuroscience \& Biobehavioral Reviews, 36(2), 747-756.

${ }^{x v}$ Sakaki, M., Yoo, H. J., Nga, L., Lee, T. H., Thayer, J. F., \& Mather, M. (2016). Heart rate variability is associated with amygdala functional connectivity with MPFC across younger and older adults. Neuroimage, 139, 44-52.

${ }^{x v i}$ Jennings, J. R., Sheu, L. K., Kuan, D. C. H., Manuck, S. B., \& Gianaros, P. J. (2016). Resting state connectivity of the medial prefrontal cortex covaries with individual differences in high-frequency heart rate variability. Psychophysiology, 53(4), 444454.

${ }^{x v i i}$ Hiser, J., \& Koenigs, M. (2018). The multifaceted role of the ventromedial prefrontal cortex in emotion, decision making, social cognition, and psychopathology. Biological Psychiatry, 83(8), 638-647.

xviii Griffiths, K. R., Quintana, D. S., Hermens, D. F., Spooner, C., Tsang, T. W., Clarke, S., \& Kohn, M. R. (2017). Sustained attention and heart rate variability in children and adolescents with ADHD. Biological psychology, 124, 11-20. 
${ }^{\text {xix }}$ Park, G., Van Bavel, J. J., Vasey, M. W., \& Thayer, J. F. (2013). Cardiac vagal tone predicts attentional engagement to and disengagement from fearful faces. Emotion, $13(4), 645$.

${ }^{x x}$ Geday, J., \& Gjedde, A. (2009). Attention, emotion, and deactivation of default activity in inferior medial prefrontal cortex. Brain and Cognition, 69(2), 344-352.

${ }^{x x i}$ Pradhan, B., \& Derle, S. G. (2012). Comparison of effect of Gayatri Mantra and poem chanting on digit letter substitution task. Ancient science of life, 32(2), 89.

xxii Gevirtz, R. (2013). The promise of heart rate variability biofeedback: Evidence-based applications. Biofeedback, 41(3), 110-120.

xxiii Pascoe, M., Alamri, Y., Dalrymple-Alford, J., Anderson, T., \& MacAskill, M. (2018). The symbol-digit modalities test in mild cognitive impairment: evidence from Parkinson's disease patients. European neurology, 79(3-4), 206-210.

${ }^{\text {xxiv }}$ López-Góngora, M., Querol, L., \& Escartín, A. (2015). A one-year follow-up study of the Symbol Digit Modalities Test (SDMT) and the Paced Auditory Serial Addition Test (PASAT) in relapsing-remitting multiple sclerosis: an appraisal of comparative longitudinal sensitivity. BMC neurology, 15, 40.

${ }^{x x v}$ Sheridan, L. K., Fitzgerald, H. E., Adams, K. M., Nigg, J. T., Martel, M. M., Puttler, L. I., ... \& Zucker, R. A. (2006). Normative Symbol Digit Modalities Test performance in a community-based sample. Archives of Clinical Neuropsychology, 21(1), 23-28.

${ }^{x x v i}$ Ohakado, M. (2017). A preliminary Study on the Level of Heart Coherence Achieved by Various Relaxation and Therapeutic Techniques. Journal of International Society of Life Information Science, 35(1), 17.

${ }^{x x v i i}$ Edwards, S. D. (2019). Empirical and heuristic phenomenological case study of the heartmath global coherence initiative. International Journal of Environmental Research and Public Health, 16(7), 1245.

xxviii Edwards, S. D. (2016). Heuristic psychological case study of HeartMath practice for health and physical exercise. African Journal for Physical Activity and Health Sciences (AJPHES), 22(Issue-42), 1125-1139.

xxix https://store.heartmath.com/emwavepro/

${ }^{\mathrm{xxx}} \mathrm{https} / / /$ play.google.com/store/apps/details?id=uk.co.jatra.inout\&hl=en

${ }^{x x x i}$ Lo, J. C., Sehic, E., \& Meijer, S. A. (2017). Measuring mental workload with low-cost and wearable sensors: Insights into the accuracy, obtrusiveness, and research usability of three instruments. Journal of cognitive engineering and decision making, 11(4), 323-336.

xxxii Benedict, R. H., DeLuca, J., Phillips, G., LaRocca, N., Hudson, L. D., Rudick, R., \& Multiple Sclerosis Outcome Assessments Consortium. (2017). Validity of the Symbol Digit Modalities Test as a cognition performance outcome measure for multiple sclerosis. Multiple Sclerosis Journal, 23(5), 721-733.

xxxiii Sheridan, L. K., Fitzgerald, H. E., Adams, K. M., Nigg, J. T., Martel, M. M., Puttler, L. I., ... \& Zucker, R. A. (2006). Normative Symbol Digit Modalities Test performance in a community-based sample. Archives of Clinical Neuropsychology, 21(1), 23-28.

${ }^{\text {xxxiv }}$ Pradhan, B., \& Derle, S. G. (2012). Comparison of effect of Gayatri Mantra and poem chanting on digit letter substitution task. Ancient science of life, 32(2), 89.

${ }^{x x x v}$ Paul, M., \& Garg, K. (2012). The effect of heart rate variability biofeedback on performance psychology of basketball players. Applied psychophysiology and biofeedback, 37(2), 131-144. 


\section{Acknowledgement}

The author appreciates all those who participated in the study and helped to facilitate the research process.

\section{Conflict of Interest}

The author declared no conflict of interest.

How to cite this article: Irani F. Z., Trivedi G. Y. \& Sinha N. (2021). Development and validation of humming process to increase heart rate variability \& attention for college students. International Journal of Indian Psychology, 9(1), 243-251. DIP:18.01.028/20210901, DOI:10.25215/0901.028 